\title{
A case study in model-based dynamic calibration of small strain gauge force transducers
}

\author{
Michael Kobusch ${ }^{1}$, Sascha Eichstädt ${ }^{2}$ \\ ${ }^{1}$ Physikalisch-Technische Bundesanstalt (PTB), Bundesallee 100, 38116 Braunschweig, Germany \\ ${ }^{2}$ Physikalisch-Technische Bundesanstalt (PTB), Abbestr. 2-12, 10587 Berlin, Germany
}

\begin{abstract}
Investigations of the model-based dynamic calibration of a small strain gauge force transducer of high bandwidth revealed new challenges for parameter identification. This paper discusses a more generalized mechanical model of the calibration set-up employed taking account of the transducer's connection to its mechanical environment at both ends. Based on new experimental sine and shock force data, the improved model is studied and its parameters are identified. It is shown that the proposed model is capable of linking the calibration results of both calibration methods to a much better degree. This paper is an extended version of the original contribution to the IMEKO 2015 conference in Prague, Czech Republic.
\end{abstract}

Section: RESEARCH PAPER

Keywords: dynamic modelling; parameter identification; sine force calibration; shock force calibration

Citation: Michael Kobusch, Sascha Eichstädt, A case study in model-based dynamic calibration of small strain gauge force transducers, Acta IMEKO, vol. 6 , no. 1, article 2, April 2017, identifier: IMEKO-ACTA-06 (2017)-01-02

Section Editor: Paul Regtien, The Netherlands

Received November 21, 2016; In final form November 21, 2016; Published April 2017

Copyright: (C) 2017 IMEKO. This is an open-access article distributed under the terms of the Creative Commons Attribution 3.0 License, which permits unrestricted use, distribution, and reproduction in any medium, provided the original author and source are credited

Funding: This work is part of the Joint Research Project IND09 "Traceable dynamic measurement of mechanical quantities" of the European Metrology Research Programme (EMRP). The EMRP is jointly funded by the EMRP participating countries within EURAMET and the European Union

Corresponding author: Michael Kobusch, e-mail: michael.kobusch@ptb.de

\section{INTRODUCTION}

The measurement of dynamic forces is widely used in many areas of industry. Increasing demands on measurement accuracy have to face the fact that dynamic force measurements are still purely based on static calibrations, not to mention the lack of documentary standards or commonly accepted guidelines for dynamic measurements [1]. For this reason, the establishment of traceable dynamic measurements is an important metrological topic, which was recently emphasized by a European Metrology Research Programme (EMRP) joint research project on the traceable dynamic measurement of mechanical quantities [2], [3].

In this paper, the general approach of a model-based calibration will be followed in which the dynamic behaviour of the force transducer in a given mechanical environment is described by a lumped-mass model using a series arrangement of spring-mass-damper elements. The transducer itself is characterized by model parameters that describe the dynamically relevant distribution of mass, stiffness and damping. The commonly used basic model of a force transducer employs two coupled model masses, i.e. four parameters: head mass $m_{\mathrm{H}}$, base mass $m_{\mathrm{B}}$, stiffness $k$, and damping $d$. The parameters of interest may be identified from dynamic measurements by fitting modelled and measured force data. The main purpose of the proposed approach is the characterization of the transducer's dynamic behaviour independent of the given experimental set-up, i.e. its mechanical environment or its type of dynamic force excitation, e.g. shock or sine.

The calibration devices considered in the following apply primary methods to provide traceability of the dynamic force measurement, which is principally based on the determination of the inertia force of an accelerated mass body. A laser interferometer (vibrometer) measures the time-dependent acceleration $a(t)$ of a mass $m$ (determined by weighing) which generates a reference force $F(t)=m \cdot a(t)$. Similar facilities for sinusoidal force excitation have been established at PTB [4], 
CEM [5] and NIST [6]. Calibration devices for shock excitation are available at PTB [7].

Initial experiences with the parameter identification of various strain gauge force transducers demonstrated some discrepancies between results from shock and sine force experiments that need to be explained. The analysis of shock force data showed that consistent results can be obtained for transducers that respond with strong signal ringing [7]. However, the previous parameter identification approach failed when the force transducer responded with smooth shock pulses, which is the case for transducers of high bandwidth and comparably long shock duration. Moreover, interlaboratory comparisons of model parameter estimates obtained with sine and shock force data using conventional modelling approaches revealed discrepant values, in particular, for the high-bandwidth force transducer of this case study. Therefore, the modelling proposed here for the sine and shock force experiments overcomes this discrepancy and allows the different reported values for the stiffness parameter to be explained.

\section{CHALLENGES}

In a given mechanical set-up, e.g. a dynamic measurement application or a dynamic calibration device, the elastically coupled structural mass components of the transducer as well as of its mechanical environment generate inertia forces that influence the dynamic measurement behaviour. The specific models of the dynamic calibration set-ups have to be appropriately developed to be able to unambiguously identify the parameters of the transducer under test from the measured data. First experiences obtained with strain gauge force transducers of differing design, size, weight and mechanical coupling revealed several challenges for the model-based calibration.

This case study is focused on dynamic measurements with a type U9B / $1 \mathrm{kN}$ strain gauge force transducer manufactured by HBM. The transducer is specified for a nominal force of $1 \mathrm{kN}$, a mass of about $65 \mathrm{~g}$ and is mounted with threaded rods at both ends (see Figure 1). In particular, this small transducer demonstrated, with its large bandwidth as well as its resonance behaviour, that the standard sine and shock force calibration set-ups have to be modified to obtain the desired dynamic information. Furthermore, it showed that the formerly published model descriptions have to be extended in a more generalized fashion to be able to cover the observed behaviour of the different test conditions.

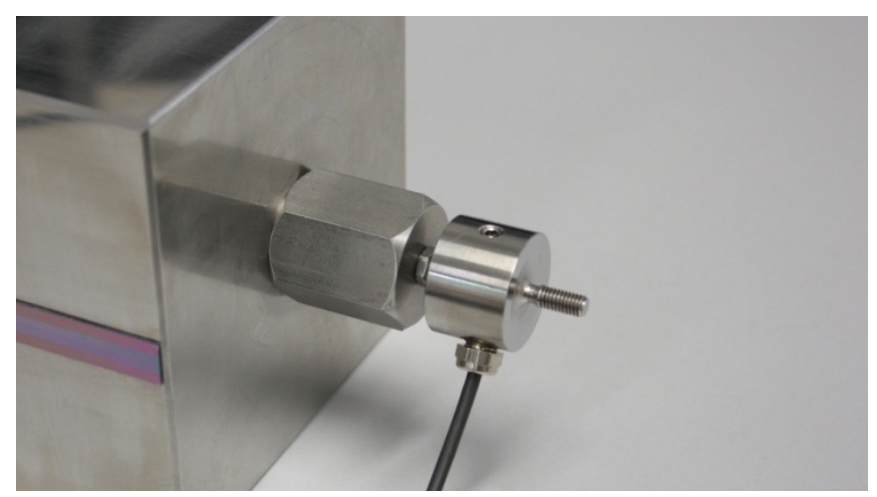

Figure 1. Force transducer HBM U9B / $1 \mathrm{kN}$ mounted at the cube-shaped $10 \mathrm{~kg}$ reaction mass of the $20 \mathrm{kN}$ shock force calibration device by means of an adapter with central threads and a counter nut.
The following challenges have been identified:

1. Previous shock force measurements showed that the dynamic models have to account for the elastic coupling at the base of the transducer [7], [8]. A weak coupling can result in a low-frequency resonance of the transducer housing which may dominate the dynamic behaviour.

2. A force transducer may also respond with more than one axial resonance depending on its structural mass distribution [9]. This behaviour was experimentally observed for the selected transducer applied to shock excitation. A modal analysis using the finite element (FE) method finally revealed that the transducer exhibits two axial resonances: a resonance of the housing at about $10 \mathrm{kHz}$, and a resonance of the upper threaded rod (head mass) at about $28 \mathrm{kHz}$ (stated values hold for an unloaded force transducer fixed at its base, cf. Figure 10).

3. The experimental shock force data may not contain sufficient dynamic information to identify the model parameters of a transducer of high bandwidth. This experience was gained with the HBM U9B / $1 \mathrm{kN}$ investigated at the $20 \mathrm{kN}$ shock force calibration device. In this case, the shock pulses are too long to substantially excite the transducer's modal vibrations.

4. The parameter identification from shock force data may be hampered by modal vibrations from the shock-excited mass bodies of the calibration device [7]. In the case of the selected transducer, the transducer exhibits a resonance frequency well above the disturbing components, so that the modal vibrations cannot be removed by low-pass filtering the measurement data for the parameter identification process.

5. For sine force calibrations, the force transducer is usually loaded with large load masses which drastically lower the system's resonance frequencies. However, with the comparably low upper frequency limit of the vibration exciters of about a few kilohertz, just one resonance could be observed to date, although the transducer possesses the above-mentioned multimode behaviour.

\section{NEW EXPERIMENTS}

To cope with the above-mentioned difficulties, several shock and sine force experiments were performed with the selected transducer using modified testing conditions. The new experiments finally achieved data better suited to the subsequent parameter identification process.

\subsection{Shock force measurements with small pendulum mass}

The measurements at the $20 \mathrm{kN}$ shock force calibration device show that pulse durations in the order of one millisecond are much too long to substantially excite modal excitations of the small HBM U9B / $1 \mathrm{kN}$.

To generate shorter pulses, the device's airborne impacting mass body of $10 \mathrm{~kg}$ was replaced by smaller pendulum masses of about $89 \mathrm{~g}$ and $7 \mathrm{~g}$, respectively [9]. Figure 2 compares the two shock pulses achieved with an impact mass of $10 \mathrm{~kg}$ and a pendulum mass of $7 \mathrm{~g}$. The former pulse width of $1.2 \mathrm{~ms}$ drops to less than $0.1 \mathrm{~ms}$ with the pendulum. It is seen that modal vibrations are excited to a much greater extent for shorter pulses.

\subsection{Shock force measurements with additional load mass}

Another method to strongly excite the modal vibrations of the small force transducer is the application of additional load masses which increase the transducer's effective head mass and shift the resonances towards lower frequency values [9]. 

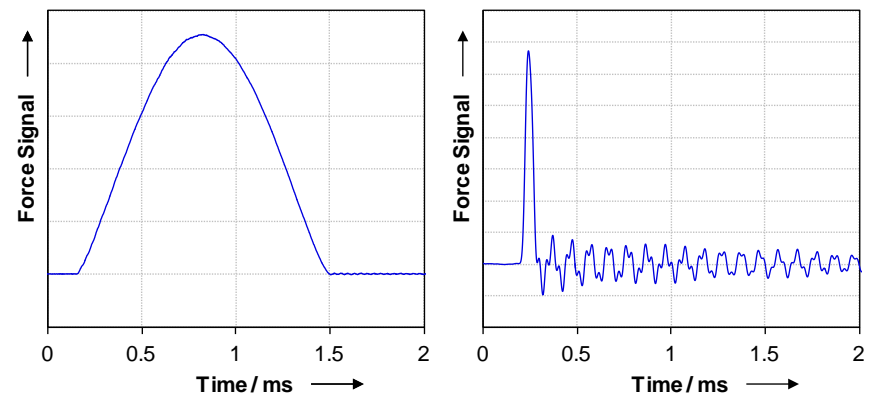

Figure 2. Shock response of the force transducer HBM U9B / $1 \mathrm{kN}$ using different impact masses, $10 \mathrm{~kg}$ (left), $7 \mathrm{~g}$ (right).

Figure 3 shows the transducer during a shock force experiment at the $20 \mathrm{kN}$ shock force calibration device using an impacting pendulum mass of $7 \mathrm{~g}$ and an additional load mass made of brass which is fixed at the free upper rod end at which the impact force is introduced. With direct access to the transducer's rod end, the available rod length somehow limits the maximum load mass value. The shock force experiments were conducted with load mass values ranging from $0.7 \mathrm{~g}$ (nut) to $18.6 \mathrm{~g}$ (cylindrical body with central thread).

Shock measurements with varied additional load mass are presented in Figure 4. The diagrams show the discrete Fourier transform (DFT) of the impact-excited signal ringing of the acceleration $a_{\mathrm{MB} 2}$ of the $10 \mathrm{~kg}$ reaction mass body and of the transducer output signal. The data shows two load-dependent resonances, the first at about $10 \mathrm{kHz}$, and the second coming down from $28 \mathrm{kHz}$, as well as several fixed resonances due to vibrational modes of the coupled mechanical environment, i.e. of the reaction mass body and the adapter.

The diagrams demonstrate that it is difficult to extract the correct resonance frequencies for the parameter identification process due to the complexity of the resonance behaviour so far not explained by the applied models. To investigate the effect of the increase of the transducer head mass, the variation of the additional load mass had to be kept small in order not to get confused with the disturbing fixed resonances.

Shock experiments with varying pulse magnitude show that the width of the shock pulses generally slightly decreases with increasing magnitude, i.e. the spectral content of the shock excitation changes accordingly. However, the spectral content of the post-impact signal ringing is almost identical for repetitive measurements even with different shock amplitudes.

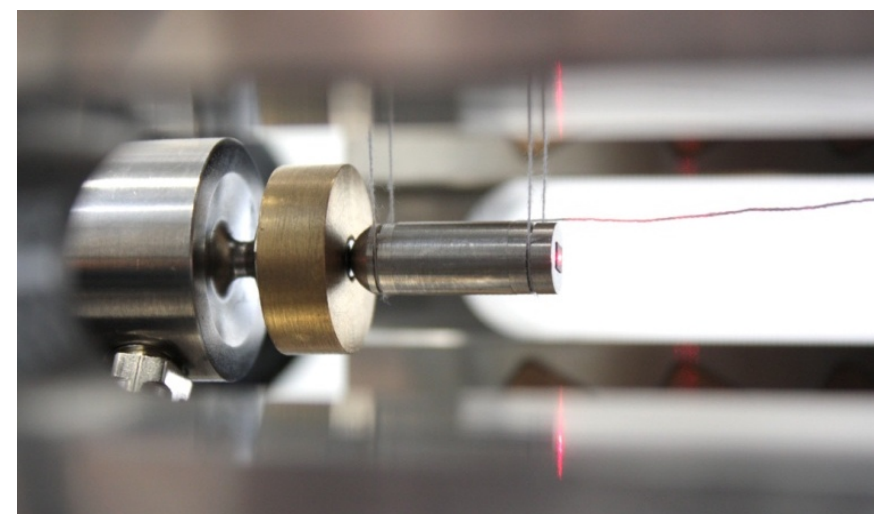

Figure 3. Shock force tests at the $20 \mathrm{kN}$ shock force calibration device using a small pendulum and additional load masses. The photo shows the $7 \mathrm{~g}$ pendulum and a load mass made of brass fastened by a counter nut (total mass of $18.6 \mathrm{~g}$ ).
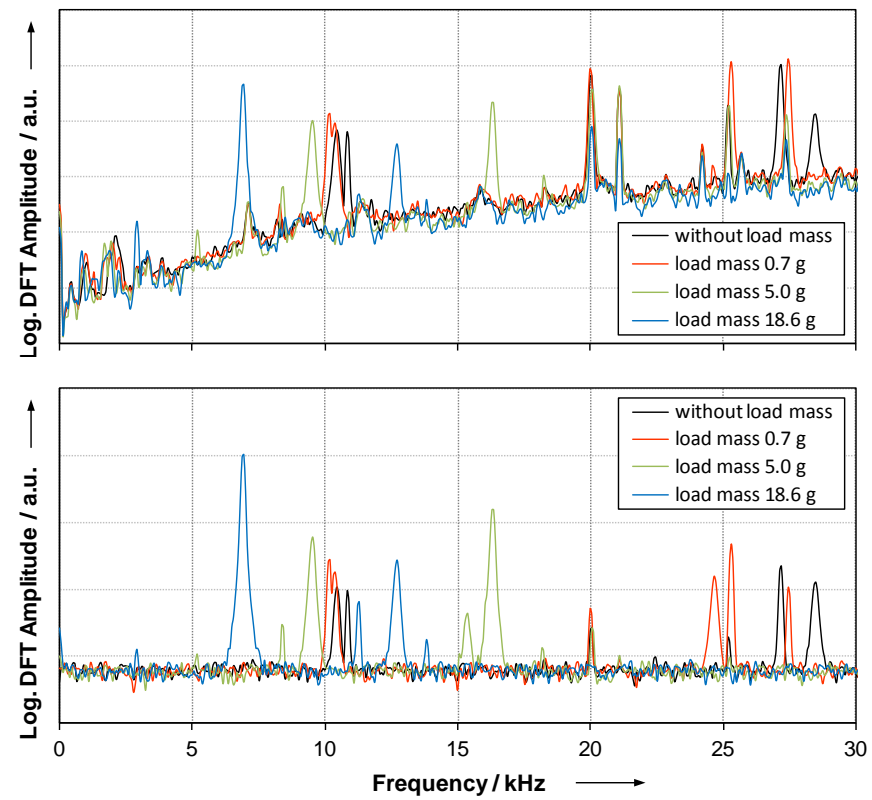

Figure 4. DFT of the shock-excited ringing of acceleration $a_{\mathrm{MB} 2}$ (top) and transducer output (bottom) obtained with different additional load masses measured at the $20 \mathrm{kN}$ shock force calibration device, shock excitation with pendulum mass of $7 \mathrm{~g}$, spectral resolution $64 \mathrm{~Hz}$, averaged signals (mean of 10).

In contrast to this excellent repeatability, the reproducibility of measurements is considerably worse. Each reassembly of the mechanical set-up usually leads to some variations of the spectral content. The resonance behaviour of the mounted transducer with attached load mass is apparently quite sensitive to the mounting conditions. The axial spacing of the components, their rotational orientation in case of asymmetries, as well as the applied fastening torque and thread lubrication might have some influence.

For instance, Figure 5 visualizes the influence of the mounting torque applied to the counter nut that fastens the transducer's base rod to the adapter (cf. Figure 1) for the four load conditions of Figure 4. The first two axial resonances identified in the DFT spectrums of the signal ringing of the transducer output are plotted in dependence of mounting torque. The upper resonance caused by the vibration of the transducer head mass (including the additional load mass) does not show any dependency as expected. However, the lower resonance representing the vibration of the transducer housing
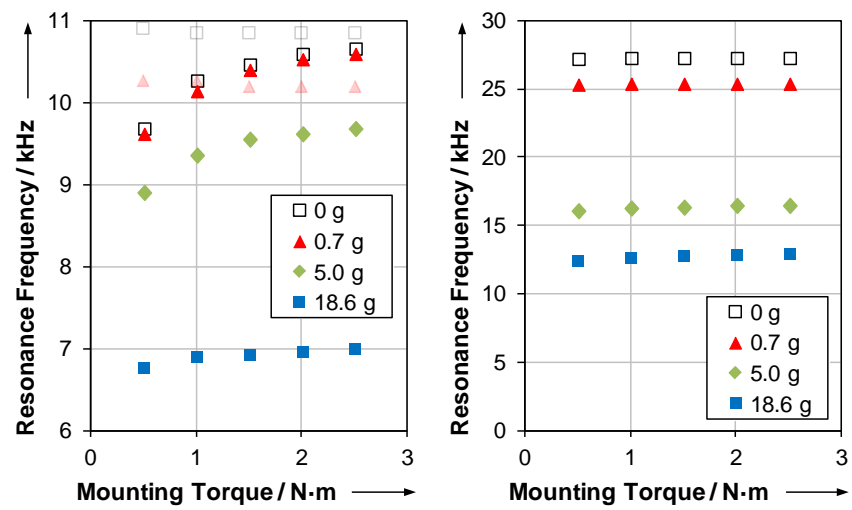

Figure 5. Resonances identified in the ringing of transducer output signal for different additional load masses and mounting torque at the transducer base, first resonance (left), second resonance (right). 
is clearly influenced by the fastening of the transducer. Higher mounting torque yields a stiffer thread connection resulting in a higher resonance frequency. For the smallest loads of $0 \mathrm{~g}$ and $0.7 \mathrm{~g}$, the first axial resonance at about $10 \mathrm{kHz}$ is difficult to be identified unambiguously as the signal ringing shows two frequency components of similar magnitude (indicated in Figure 5 by pale symbols).

Shock force tests with additional head mass obtained data better suited to the parameter identification process, but it actually proved that this transducer is more complex and its model description has to be refined. For more clarity of the modal structure and also for the purpose of understanding the sine and shock excitation responses, experimental tests with varied load mass were also performed with sine excitation using a high-frequency shaker system.

\subsection{Sine force measurements at the HF vibration exciter}

Sine force calibrations were carried out at the high-frequency (HF) acceleration standard measuring device of PTB in order to confirm the modal behaviour observed in the shock force experiments. Furthermore, it was necessary to gain experience with small load masses to fully understand the dynamic behaviour and to explain the discrepant values of the parameter identification so far observed in the different experiments.

Figure 6 shows the force transducer HBM U9B / $1 \mathrm{kN}$ with an additional load mass mounted at the platform of the HF shaker. Two laser vibrometers measure the reference acceleration of the base picked up at the upper surface of the applied adapter which connects the force transducer to the moving ceramic armature of the shaker. A new set of load masses made of brass with 14 logarithmically graded values from $0.3 \mathrm{~g}$ to $215 \mathrm{~g}$ was used for the sine force experiments. The mass bodies have a central thread of about $4 \mathrm{~mm}$ in length and were fixed with a counter nut $(0.7 \mathrm{~g})$ applying a controlled torque to achieve good reproducibility. For the smallest load mass value of $0.3 \mathrm{~g}$, the mass body was directly fastened to the transducer's thread run-out.

The diagrams of Figure 7 present the measured amplitude response, i.e. the amplitude ratio of the transducer output and the base acceleration, of the force transducer without and with additional load masses of greatly differing values (from $1.3 \mathrm{~g}$ up to $216 \mathrm{~g}$ ). The measurements clearly show two resonances, which excellently confirm the behaviour seen in the shock force experiments. The upper diagram demonstrates that a large load mass predominantly affects the lower resonance, whereas a very small mass has the greatest influence on the higher resonance.

For sine force calibrations, the force transducer is usually

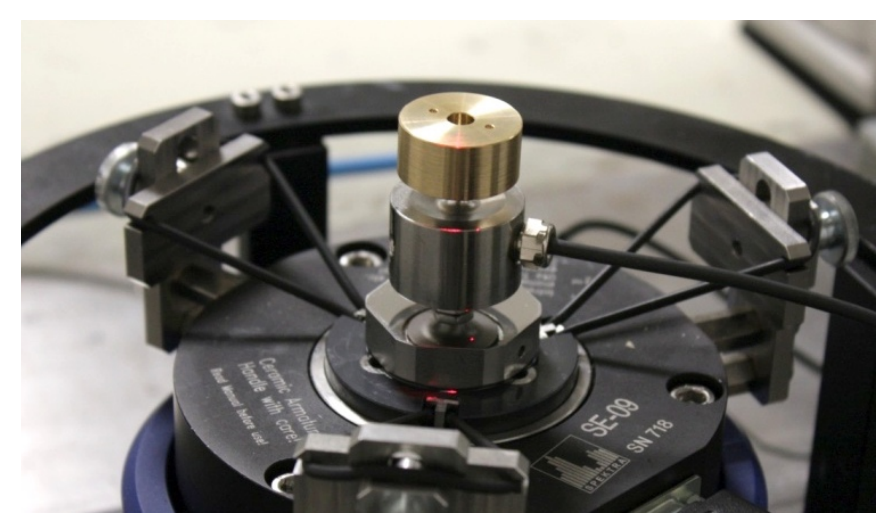

Figure 6. High-frequency sine force tests with additional load masses at the $\mathrm{HF}$ acceleration standard measuring device.
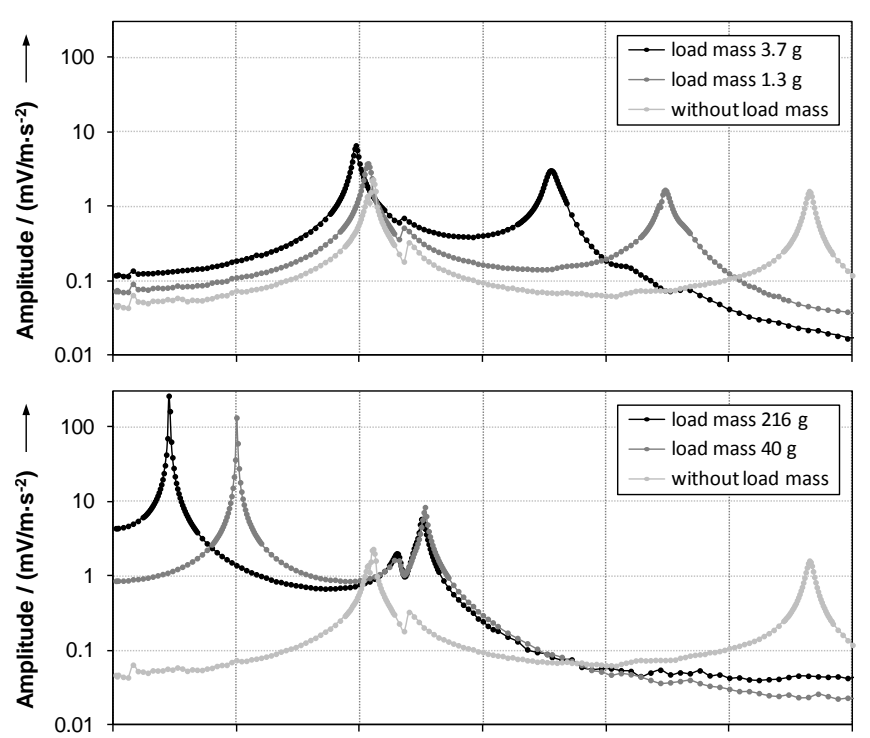

Figure 7. Amplitude response (ratio of transducer output and base acceleration) of the HBM U9B / $1 \mathrm{kN}$ obtained with different load masses measured at the HF acceleration standard measuring device.

loaded with large load masses which drastically lower the system's resonance frequencies. With the comparably low upper frequency limit of the vibration exciters of about a few kilohertz, just one resonance could be observed to date, although the transducer possesses the above-mentioned multimode behaviour.

\section{MODELLING AND PARAMETER IDENTIFICATION}

In the following, extended parametric lumped-mass models for sine and shock force experiments are proposed. Data from shock force experiments as well as sine force excitations will be analysed to determine the model parameters of the transducer under test and to verify the proposed model.

\subsection{Analysis of shock force measurements}

With regard to the model describing the shock force calibration, it is proposed to model the connection between the load mass (load button) and the transducer's head mass as elastically coupled. This elastic coupling was originally applied only for the sine force calibration with large load masses [4]. In contrast, sine force models have not yet considered an elastic coupling at the base to date. In order to obtain a sound understanding of the dynamic behaviour independent of the experimental set-up, the model of the force transducer requires elastic couplings at both ends of the transducer.

Figure 8 presents an extended model of the $20 \mathrm{kN}$ shock force calibration device with the mounted force transducer for which all mechanical couplings are considered as non-rigid. The

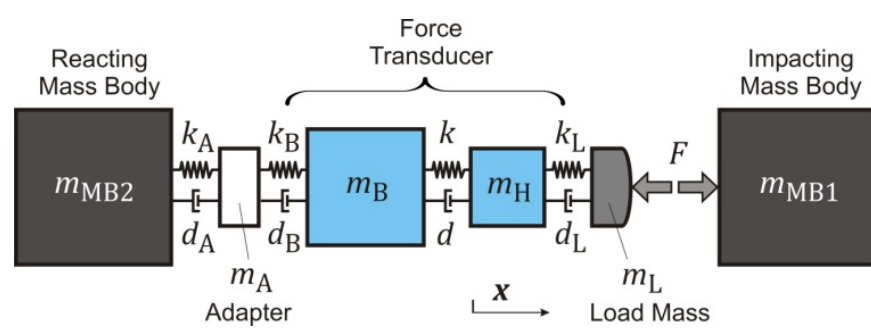

Figure 8. Model of the $20 \mathrm{kN}$ shock force calibration device with mounted force transducer and additional load mass. 
force transducer marked in blue is elastically coupled at both ends to its mechanical environment, which is the base adapter connecting the transducer to the reacting mass body, and the load mass, to which the impact force is applied. The model may be simplified if the adapter is more rigidly coupled to the reacting mass than to the transducer comparing the different mounting conditions (contact area, thread size, mounting torque), see Figure 9. This model will be considered for the shock force analysis in the remainder of this paper.

Having identified the respective resonances observed in the DFT analysis of Figure 4, the interesting stiffness parameters of the couplings and the transducer's measuring spring can be determined from the eigenvalues of the characteristic system matrix neglecting damping [7].

In general, the dynamic behaviour of the model components is described by a system of linear ordinary differential equations (ODE) derived from the equilibrium of forces at each mass element as

$M \ddot{x}+D \dot{x}+K x=L$,

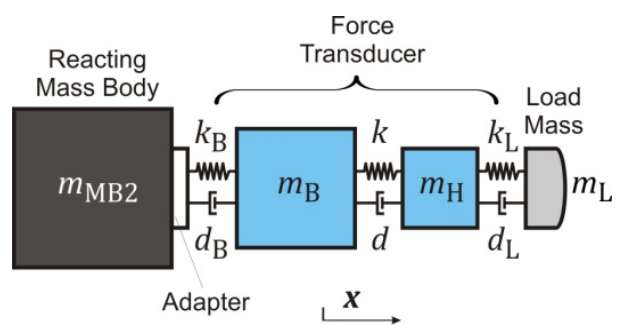

Figure 9. Series arrangement of four model masses to describe the resonance frequencies of the $20 \mathrm{kN}$ shock force calibration device. where $\boldsymbol{x}, \dot{\boldsymbol{x}}$ and $\ddot{\boldsymbol{x}}$ are the motion vectors (displacement, velocity, acceleration), $\boldsymbol{M}, \boldsymbol{D}$, and $\boldsymbol{K}$ denote the matrices for mass, damping and stiffness, and $\boldsymbol{L}$ is the load vector.

Neglecting damping, the different resonance frequencies are calculated from the eigenvalues of the characteristic system matrix.

$f_{\text {Res }}=\frac{1}{2 \pi} \sqrt{\operatorname{eig}\left(M^{-1} K\right)}$

Nevertheless, one has to keep in mind that the chosen uniaxial lumped-mass models can principally neither model bending modes nor elastic modes of three-dimensional bodies. This means that some of the observed resonances (cf. Figure 4) may not be described by the model, such as the fixed resonances at higher frequencies which are attributed to elastic modes of the cube-shaped reacting mass body of $10 \mathrm{~kg}$.

More detailed information on the modal resonances was obtained by FE simulations of the conducted shock force experiments. To consider the observed dependencies on the mounting conditions, the variable stiffness of the transducer's thread connections was approximated by a simplified thread model consisting of an inner core of full rigidity surrounded by an outer shell with reduced values for the Young's modulus and the shear modulus, as well as with reduced density to obtain a correct mass value. The calculated simulations did not consider the electronic components, the sealing, and the cables which are elastically fixed inside the transducer housing, as these parts of low mass and low stiffness should not considerably influence the dynamic behaviour of the firmly connected metallic parts.

Furthermore, the cable connector was modelled as rigidly fixed to ease the interpretation of the simulated modal shapes. Figure 10 visualizes the simulated modal shapes (obtained with Autodesk Inventor) of the first two axial resonances for the
Load Mass:

$0 \mathrm{~g}$
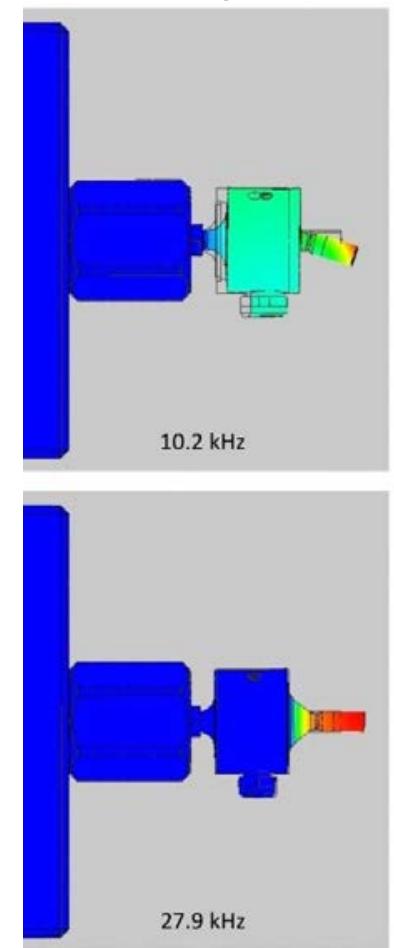

$0.7 \mathrm{~g}$
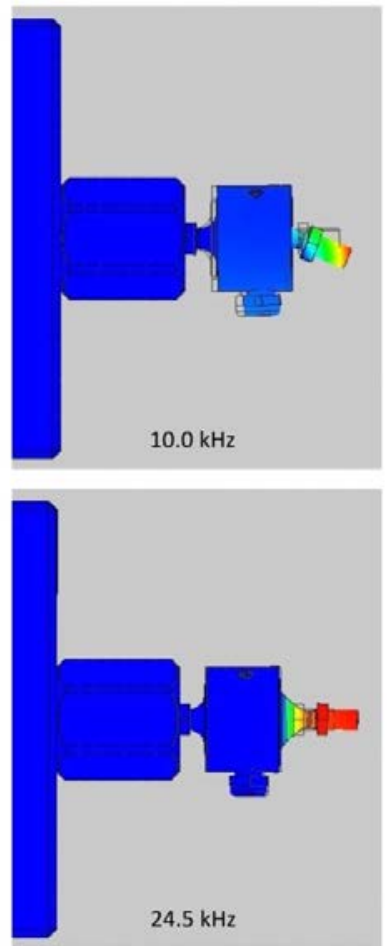

$5.0 \mathrm{~g}$
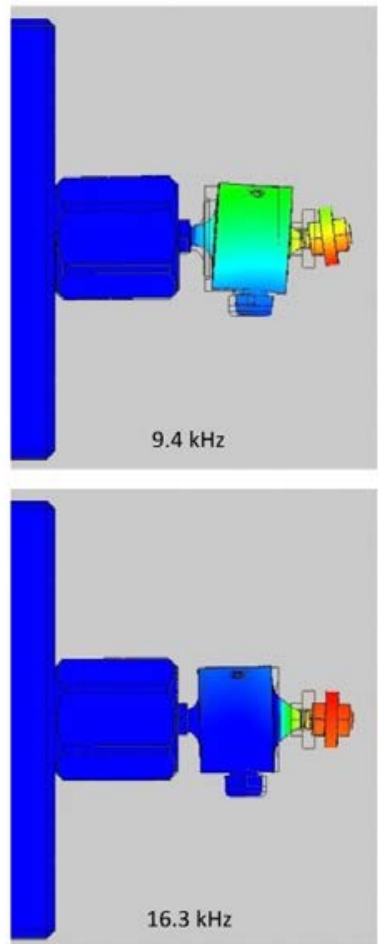

$18.6 \mathrm{~g}$
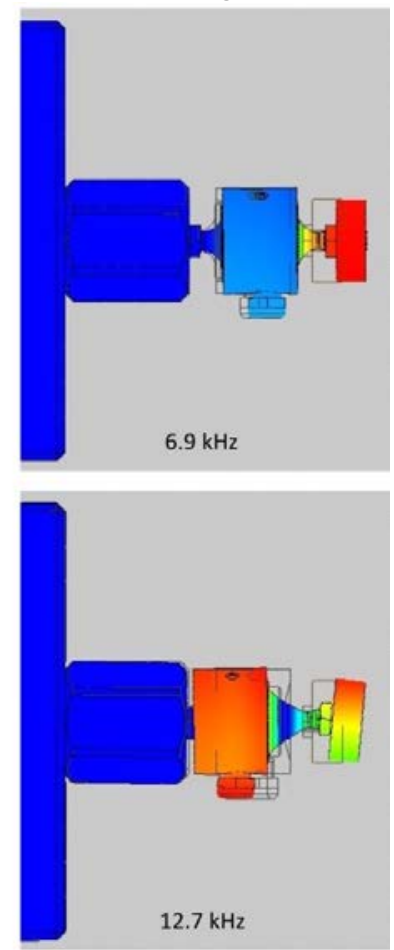

Figure 10. FE simulations of the modal shapes of the force transducer HBM U9B / $1 \mathrm{kN}$ with different load masses for shock tests at the $20 \mathrm{kN}$ shock force calibration device: first axial resonance (top row), second axial resonance (second row). The images show the deformed geometry with colour-coded elongation (auto-scaled), the undeformed geometry is outlined in grey. 
four load conditions previously considered. The upper part of the transducer (with its load mass) and the transducer housing perform an in-phase motion at the first axial elongation mode, and an out-of-phase motion at the second mode. The simulation results agree pretty well with the experimentally observed resonances (cf. Figure 4). Due to the asymmetric mechanical structure, the axial elongation modes exhibit some superposed bending, which becomes particularly pronounced in the vicinity of an explicit bending mode. The simulations further show that the first bending resonance appears at fairly low frequencies, at $1.3 \mathrm{kHz}$ for a transducer without load mass, and at $1.0 \mathrm{kHz}$ for $m_{\mathrm{L}}=18.6 \mathrm{~g}$. Depending on the applied load mass, even the second or third bending mode may appear before the first axial mode. However, the force transducer output did not show these modes in the conducted shock force experiments with the exception of the first bending mode of the upper threaded bolt (at $10.6 \mathrm{kHz}$ without load mass) that may almost coincide with the transducer's first axial resonance (cf. Figure 5 left).

The influence of elastic modes of the reacting mass body is visualized in Figure 11, which presents some selected modes of interest. The first torsion-type mode of the mass body appears at about $13.2 \mathrm{kHz}$. It does, however, not affect the vertical measuring axis and thus merely excites the force transducer. In contrast, the two modal resonances at about $20 \mathrm{kHz}$ will affect the on-axis vibrometer measurement, in which the mode at $19.8 \mathrm{kHz}$ also affects the transducer, but the $20.8 \mathrm{kHz}$ mode does not. This result is in excellent agreement with the previously presented measured shock spectra (cf. Figure 5). The simulations also give explanations for other observed fixed resonances at higher frequencies.

\subsection{Analysis of sine force measurements}

Regarding the mechanical set-up for sine force calibrations, the proposed model takes the form depicted in Figure 12.

The vibration exciter generates a sinusoidal acceleration $\ddot{x}_{\mathrm{S}}$ of the shaker platform which introduces a sinusoidal input force $F_{\mathrm{S}}$ at the base of the elastically coupled force transducer given by

$F_{\mathrm{S}}=d_{\mathrm{B}} \dot{x}_{\mathrm{S}}+k_{\mathrm{B}} x_{\mathrm{S}}$.

The mechanical model of the sine force calibration set-up is once again described by the ODE system (1), whose components take the following forms.

$\boldsymbol{M}=\left[\begin{array}{ccc}m_{\mathrm{L}} & 0 & 0 \\ 0 & m_{\mathrm{H}} & 0 \\ 0 & 0 & m_{\mathrm{B}}\end{array}\right]$

$\boldsymbol{D}=\left[\begin{array}{ccc}d_{\mathrm{L}} & -d_{\mathrm{L}} & 0 \\ -d_{\mathrm{L}} & d_{\mathrm{L}}+d & -d \\ 0 & -d & d+d_{\mathrm{B}}\end{array}\right]$

$\boldsymbol{K}=\left[\begin{array}{ccc}k_{\mathrm{L}} & -k_{\mathrm{L}} & 0 \\ -k_{\mathrm{L}} & k_{\mathrm{L}}+k & -k \\ 0 & -k & k+k_{\mathrm{B}}\end{array}\right]$

$\boldsymbol{x}=\left[x_{\mathrm{L}}, x_{\mathrm{H}}, x_{\mathrm{B}}\right]^{\mathrm{T}}$

$\boldsymbol{L}=\left[\begin{array}{c}0 \\ 0 \\ d_{\mathrm{B}} \dot{x}_{\mathrm{S}}+k_{\mathrm{B}} x_{\mathrm{S}}\end{array}\right]$

The output signal $U_{\mathrm{F}}$ of the force transducer is proportional to the elongation of the transducer's measuring spring $k$ and is given by
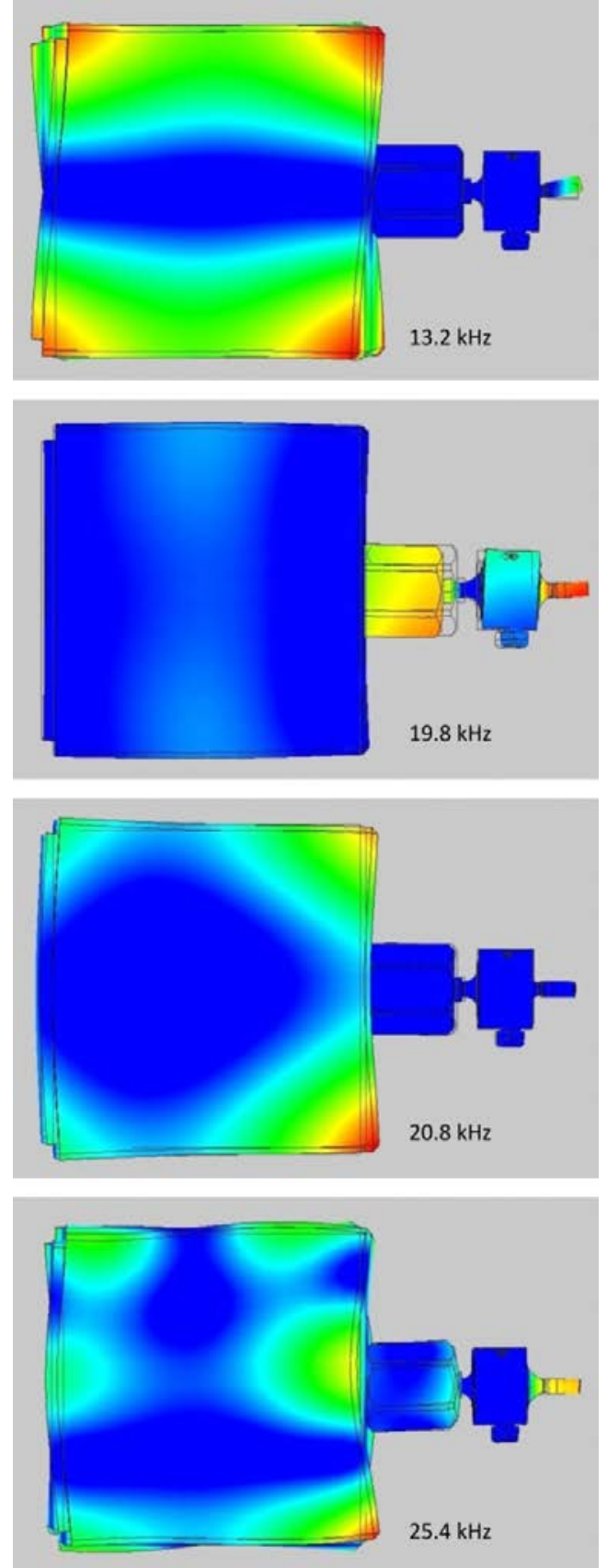

Figure 11. FE simulations of the modal shapes of the $10 \mathrm{~kg}$ reacting mass body when shock testing the HBM U9B /1 kN without load mass.

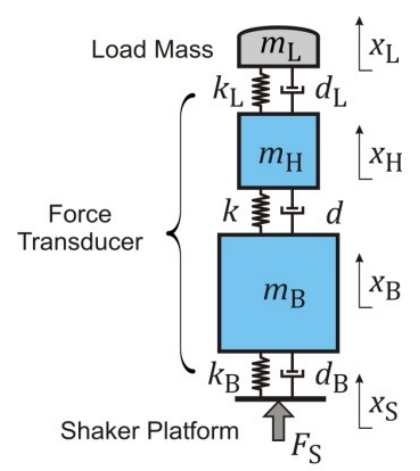

Figure 12. Model of the sine force calibration device with mounted force transducer and additional load mass. 
$U_{\mathrm{F}}=\rho\left(x_{\mathrm{H}}-x_{\mathrm{B}}\right)$,

where $\rho$ denotes a scaling factor.

The sought transfer function of the force signal $U_{\mathrm{F}}$ and acceleration $\ddot{x}_{\mathrm{S}}$ is expressed in its Laplace transform as

$H_{\mathrm{FS}}(s)=\frac{U_{\mathrm{F}}(\mathrm{s})}{\ddot{x}_{\mathrm{S}}(\mathrm{s})}=\frac{\rho\left(x_{\mathrm{B}}-x_{\mathrm{H}}\right)}{s^{2} x_{\mathrm{S}}}=\frac{\rho\left(z_{\mathrm{B}}-z_{\mathrm{H}}\right)}{s^{2}}$

For neglected damping, equation (6) becomes

$$
\begin{aligned}
H_{\mathrm{FS}} \text { undamped } & (s)=\frac{A(s)}{B(s)}, \\
A(s)= & \rho k_{\mathrm{B}}\left(m_{\mathrm{H}}+m_{\mathrm{L}}\right)\left[k_{\mathrm{L}}+\frac{m_{\mathrm{H}} m_{\mathrm{L}}}{m_{\mathrm{H}}+m_{\mathrm{L}}} s^{2}\right], \\
B(s)= & -k^{2}\left(k_{\mathrm{L}}+m_{\mathrm{L}} s^{2}\right)+\left[k+k_{\mathrm{B}}+m_{\mathrm{B}} s^{2}\right] \times \\
& {\left[-k_{\mathrm{L}}^{2}+\left(k+k_{\mathrm{L}}+m_{\mathrm{H}} s^{2}\right)\left(k_{\mathrm{L}}+m_{\mathrm{L}} s^{2}\right)\right] . }
\end{aligned}
$$

Figure 13 presents a comparison of the amplitude responses of the measured and fitted models for four greatly differing load masses (about $216 \mathrm{~g}, 27 \mathrm{~g}, 6.7 \mathrm{~g}$ and $0.3 \mathrm{~g}$ ). The fitting process applied different weighting procedures: no weighting and weighting with emphasis on a frequency interval of a width of $1 \mathrm{kHz}$ centred on the respective resonance frequency using the SciPy implementation of the sequential quadratic programming constrained optimization routine [10]. It is seen that the fitting result is sensitive to the chosen weighting scheme in the fitting method. For excitations above $20 \mathrm{kHz}$, the measured signal significantly deviates from the model responses as measurement noise becomes more dominant. Assuming the transducer stiffness to be constant in all experiments, an estimate of its value could be derived from analysing fit results for all available data from sinusoidal experiments. However, for load masses greater than $10 \mathrm{~g}$, a parasitic resonance at about $12 \mathrm{kHz}$ affects the amplitude response in the vicinity of the second resonance. To explain the experimentally observed parasitic resonance, the sine force experiments were investigated by FE methods using ANSYS Workbench. Here, the moving mass of the shaker was simplified by a cylindrical ceramic body of $230 \mathrm{~g}$; the force transducer was modelled as previously described (cf. Section 4.1), taking into account the coupling stiffness of the transducer's threaded connections. The modelled harmonic response represents the flexing of the transducer's structural spring element, calculated as the averaged deformation in measuring direction of the ring-shaped interior surface applied with the sensing strain gauges, related to the acceleration of the adapter surface, which is the reference signal for the measurements.

As an example, Figure 14 compares the measured and modelled amplitude response of the force transducer HBM U9B / $1 \mathrm{kN}$ loaded with a mass of $41 \mathrm{~g}$. The measured and modelled frequency responses agree quite well and show larger deviations only at the upper resonance with its disturbing double-peak structure. In the figure, the solid black line is the frequency response of the measurement set-up without any motion constraints, the dashed line the response of a transducer housing which would be axially guided. The latter model simulates the behaviour of an axisymmetric transducer, where inertia forces (e.g. from the cable connector) could not excite tilting modes, demonstrating that this structure would respond with a single upper resonance. In the case of the free unconstrained model, the program calculated five modal
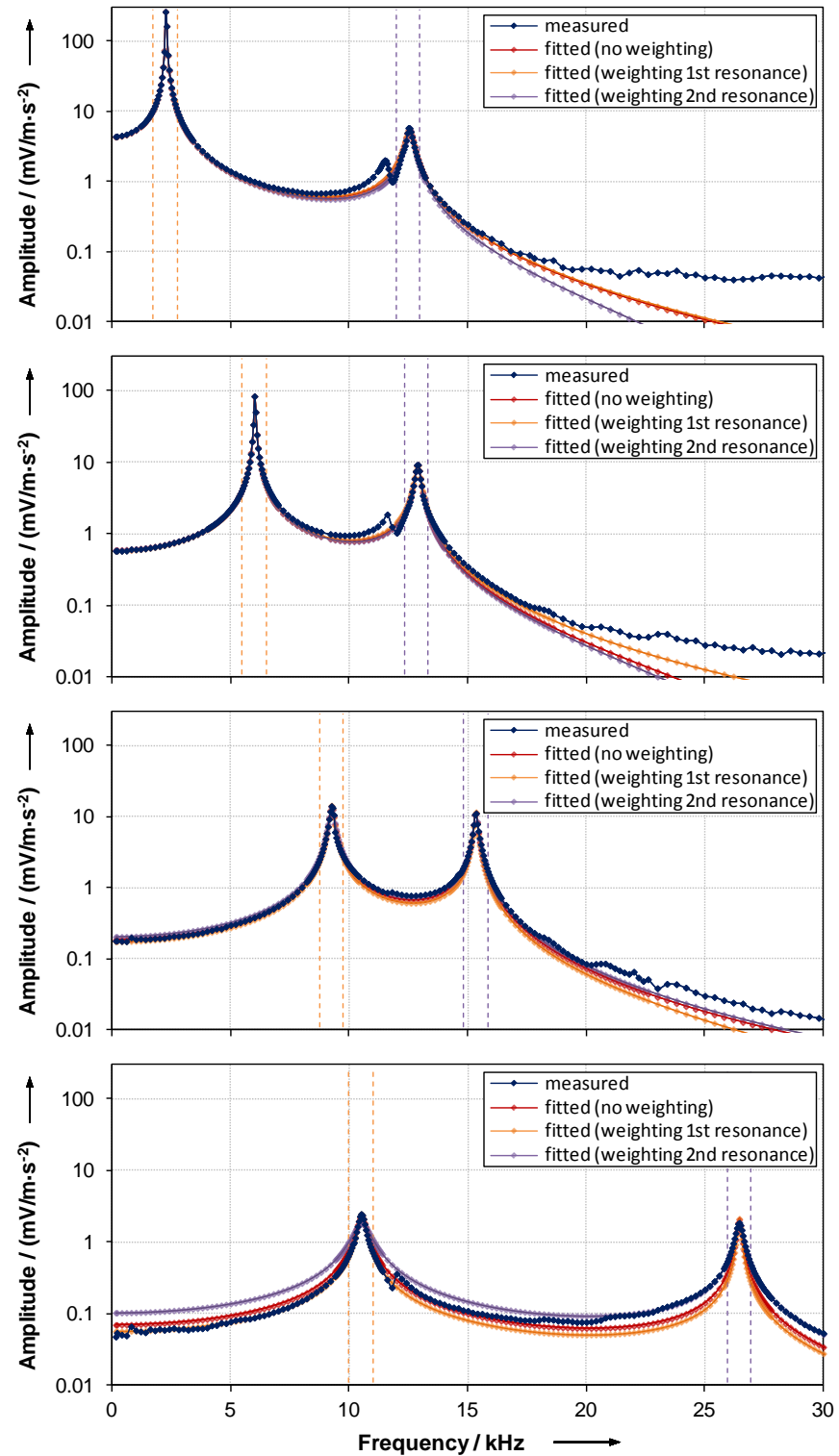

Figure 13. Comparison of fitting procedures for the parameter identification from sine force measurements using the force transducer HBM U9B / $1 \mathrm{kN}$ applied with different load masses (from top to bottom $216 \mathrm{~g}, 27 \mathrm{~g}, 6.7 \mathrm{~g}$ and $0.3 \mathrm{~g})$.

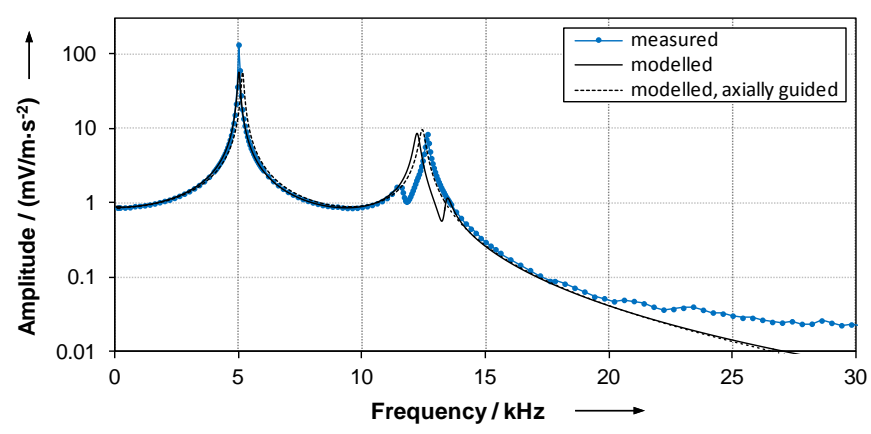

Figure 14. Measured and FE modelled amplitude response of sine force measurements of the HBM U9B / $1 \mathrm{kN}$ applied with a load mass of $41 \mathrm{~g}$.

resonances in the considered frequency range from $11 \mathrm{kHz}$ to $14 \mathrm{kHz}$. 
Respective harmonic analyses of sine force measurements using other load masses confirm the finding that the observed parasitic resonance is caused by tilting modes of the transducer housing. Further refinement of the FE modelling seems impracticable as it would probably require detailed knowledge of the transducer's interior, e.g. the strain gauge positions and the circuitry, or the modelling of cable and sealing compounds.

To mitigate the unavoidable influence of the parasitic resonance on the estimated model stiffness parameters, a fit of the stiffness matrix $\boldsymbol{K}$ was performed, so that the location of the resonances for a range of load masses can be expressed by the model. Therefore, resonance frequencies were extracted from the sine force measurements with load masses ranging from $0 \mathrm{~g}$ to about $216 \mathrm{~g}$. Uncertainties of $50 \mathrm{~Hz}$ were associated with those readings, except for the second resonance for load masses greater than $10 \mathrm{~g}$, where larger values of $200 \mathrm{~Hz}$ were chosen to account for the influence of the parasitic resonance. A Bayesian regression [11] was applied to the resonance frequencies with the above uncertainties. As the prior distributions for the model stiffness parameters, independent normal distributions $N\left(\mu, \sigma^{2}\right)$ with the mean $\mu$ and the standard deviation $\sigma$ were applied with $k_{\mathrm{L}} \sim N\left(3.4 \cdot 10^{8}\right.$, $\left.\left(5 \cdot 10^{7}\right)^{2}\right) \mathrm{N} / \mathrm{m}, k \sim N\left(7.1 \cdot 10^{7},\left(1 \cdot 10^{7}\right)^{2}\right) \mathrm{N} / \mathrm{m}$ and $k_{\mathrm{B}} \sim N\left(2.7 \cdot 10^{8}\right.$, $\left.\left(1 \cdot 10^{7}\right)^{2}\right) \mathrm{N} / \mathrm{m}$ which were derived from investigating various experiments with the same force transducer. The resulting marginal posteriors of the three stiffness parameters are visualized in Figure 15. The values obtained were found to be approximately normally distributed, giving a transducer stiffness with a mean value of $k=7.03 \cdot 10^{7} \mathrm{~N} / \mathrm{m}$ and a standard deviation $\sigma_{k}$ of $1.5 \cdot 10^{5} \mathrm{~N} / \mathrm{m}$. Note that this is an initial regression analysis using only the location of the measured resonances from the sinusoidal data and model equation (2). Therefore, the obtained posterior standard deviations are not the uncertainties associated with the three stiffness parameters sought, but are rather an indication of the trustworthiness of their estimates given the prior information applied and using model equation (2). A full regression analysis would require

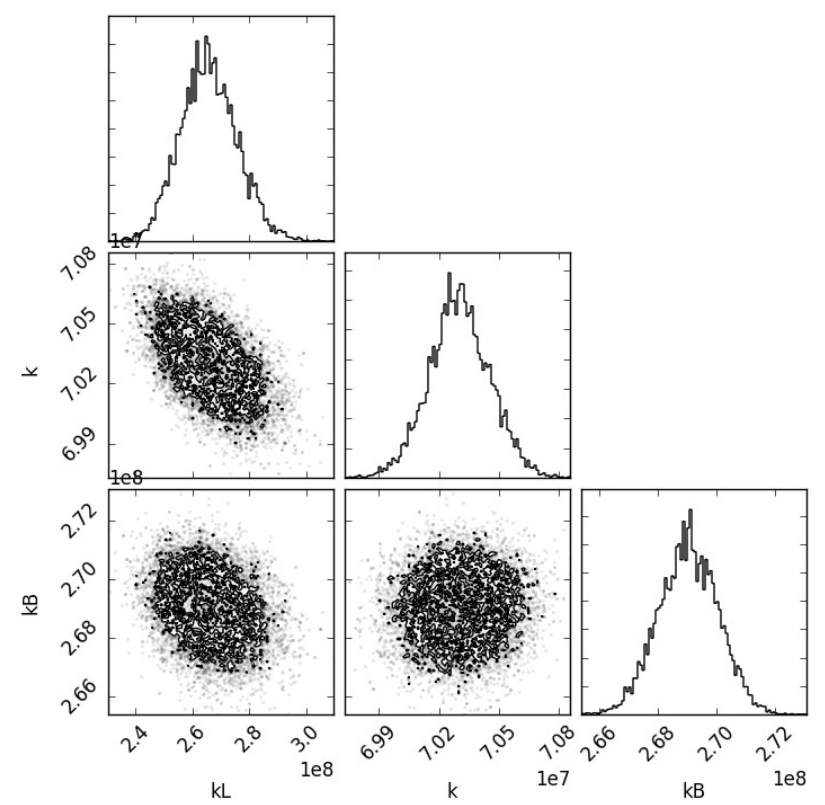

Figure 15. Stiffness parameters $k, k$ and $k$ B resulting from a Bayesian regression applying resonance frequency values extracted from sine force measurements with load masses ranging from $0 \mathrm{~g}$ to about $216 \mathrm{~g}$. defining a statistical model based on model equation (4) for the different data sets and measurement set-ups, which is beyond the scope of this paper.

Future research will focus on the optimization of the presented parameter identification and the employed fitting methods, in particular, on how to treat the influence of the parasitic resonances of the transducer's tilting modes. For instance, a simultaneous fitting of measurements obtained with different load masses together with an appropriate statistical model for the set of measurements may achieve an improved parameter estimation. The insights gained from such investigations can be expected to improve the understanding of the calibration of force transducers with huge bandwidth using sine or shock excitation measurements, respectively.

\subsection{Comparison and discussion}

Figure 16 compares measured and modelled resonance frequencies of the HBM U9B / $1 \mathrm{kN}$ applied with different load masses. The diagram visualizes the three modelled resonances in dependence on the load mass. It is seen that the highest resonance stays well above $60 \mathrm{kHz}$ and thus cannot be observed experimentally. The lowest resonance - the vibration of the transducer housing - starts at about $10 \mathrm{kHz}$ and significantly decreases with increasing mass for loads greater than about $10 \mathrm{~g}$. The second resonance starts at $28 \mathrm{kHz}$ and drops to about $11 \mathrm{kHz}$ for increasing loads.

The figure also displays the resonance frequency calculated for a model of only one degree of freedom (dotted line) with a stiffness identical to the total stiffness of the series arrangement of the coupled mechanical components separately described by models of higher degrees of freedom. It is seen that the model of one degree of freedom significantly deviates for smaller load masses. That is, the simple model is insufficient to model the full range of load masses and to agree with parameter identifications with small or without load masses, for example, from shock force experiments.

The diagram further plots the resonant frequencies which were observed during various shock (black crosses) and sine force (red circles) experiments. The red square represents the sine force measurement performed at the Centro Español de

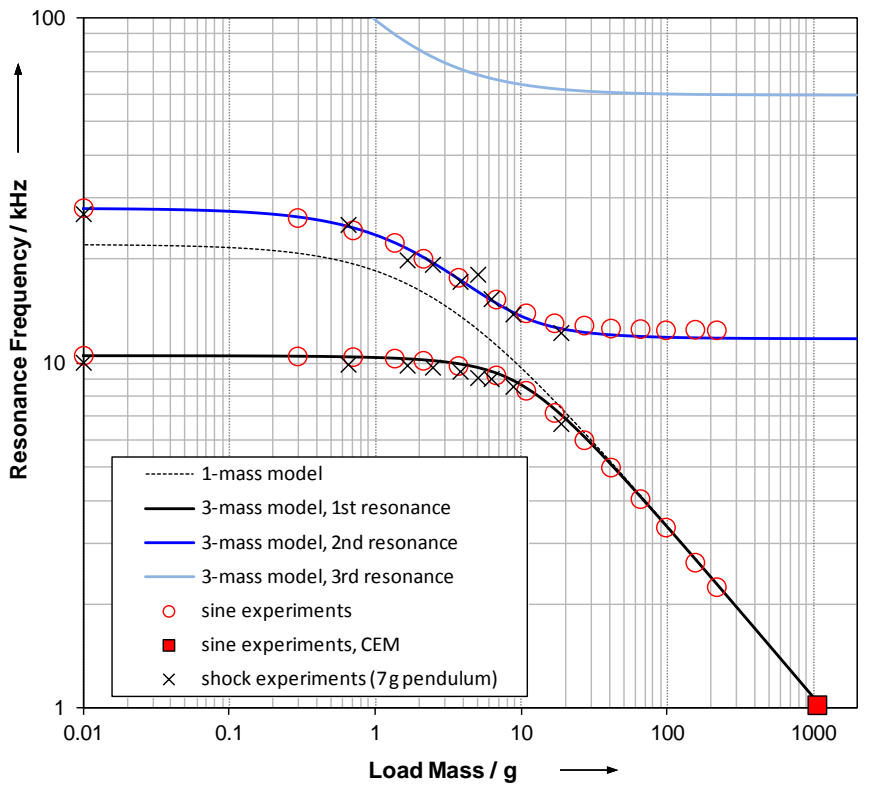

Figure 16. Comparison of measured and modelled resonance frequencies of the HBM U9B / $1 \mathrm{kN}$ with different load masses. 
Metrología (CEM) using a large load mass of $1 \mathrm{~kg}$ [12].

The measured values from the different shock and sine force experiments agree well with the simulated values given by the proposed model with three degrees of freedom. Some larger deviations related to the second resonance at higher loads may be caused by the parasitic tilting modes previously described. In this context it has to be noted that the experimentally measured resonances generally belonged to two basically differing measurement set-ups with partly different mechanical components and differing mounting conditions, which may further explain the deviations of the observed resonance frequencies.

The good agreement of the modelled resonance frequencies demonstrates the advantage of the proposed parametric calibration approach. That is to say, in principle the parameter values associated with the force transducer model can be applied in measurements which are of a different nature to the calibration.

This statement is further illustrated in the following Figures 17 to 19 , where we applied the transducer stiffness parameter of $k=7.03 \cdot 10^{7} \mathrm{~N} / \mathrm{m}$, which is the result of the parameter identification by a Bayesian regression for the resonance frequencies derived from the sinusoidal experiments, to the shock force model depicted in Figure 9 in order to model the transducer output of shock force calibration measurements with different impacting mass bodies and additional load masses, respectively. Therefore, the transducer stiffness was plugged into the model equations for the shock force model with two degrees of freedom, and the remaining stiffness and damping parameters of the model were adjusted accordingly.

Figure 17 compares the modelled and measured force transducer output as well as the model's force input signal $F$ for the large airborne mass body of $10 \mathrm{~kg}$ and the small pendulum of $89 \mathrm{~g}$, respectively. The input force is determined by numerical calculation of the acceleration of the impact mass from measured displacement data, where low-pass filtering was applied to reduce noise amplification. Whereas the broad force pulse (pulse width of $1.3 \mathrm{~ms}$ ) obtained with the large impacting

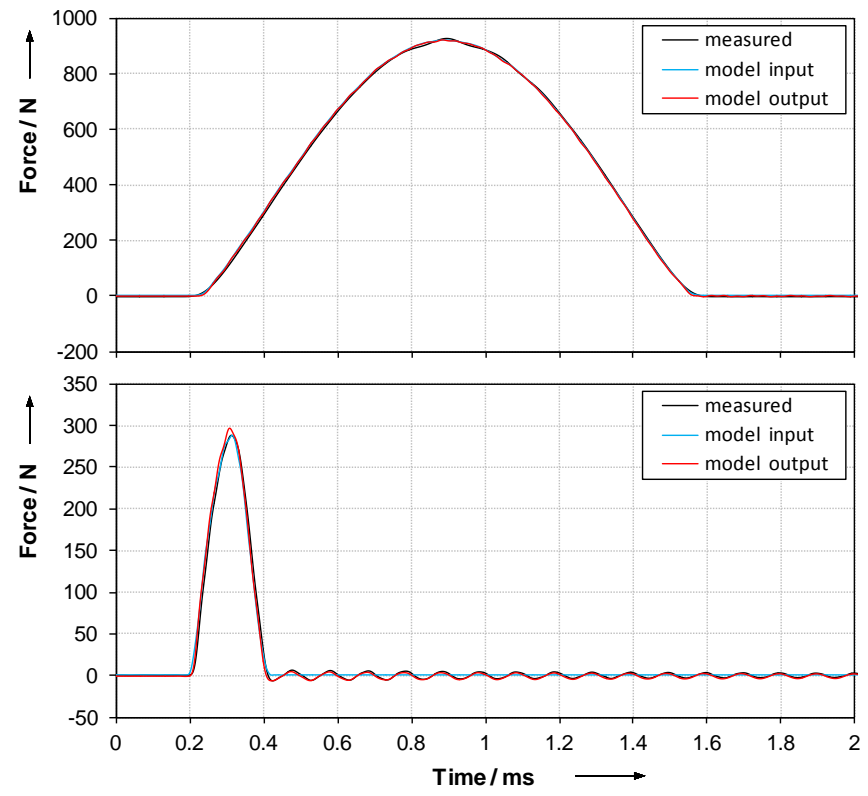

Figure 17. Measured and modelled transducer output for the shock excitation with an impacting mass of about $10 \mathrm{~kg}$ (top) and $89 \mathrm{~g}$ (bottom), transducer stiffness estimated from sinusoidal excitation measurements.
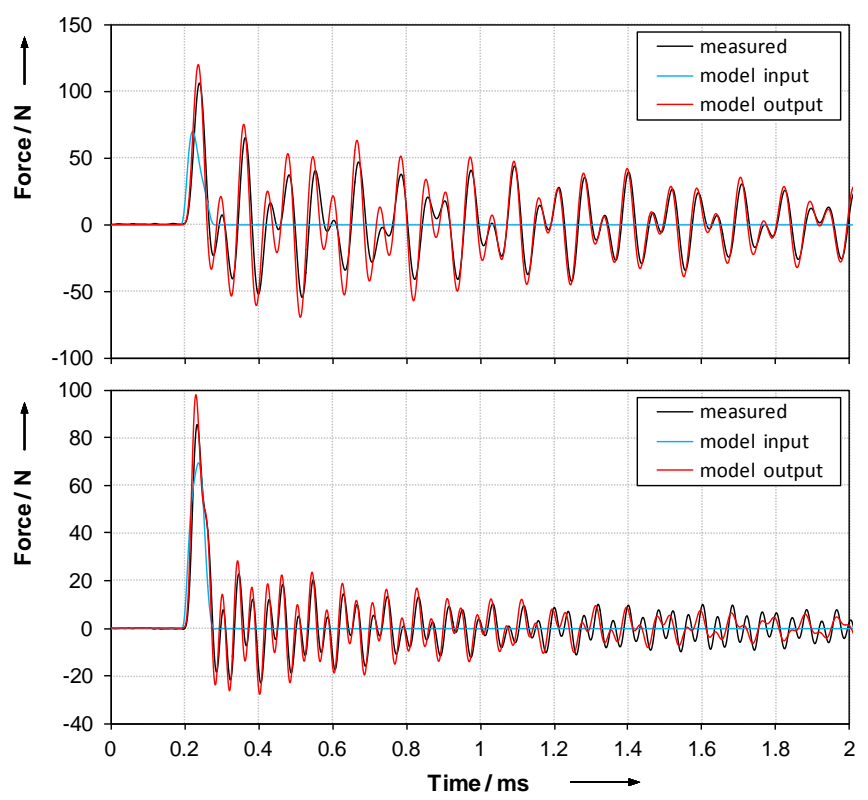

Figure 18. Comparison of measured and modelled force transducer output for a shock excitation with an impacting pendulum mass of $7 \mathrm{~g}$, load mass $5 \mathrm{~g}$ (top) and $0.65 \mathrm{~g}$ (bottom), transducer stiffness estimated from sinusoidal excitation measurements.
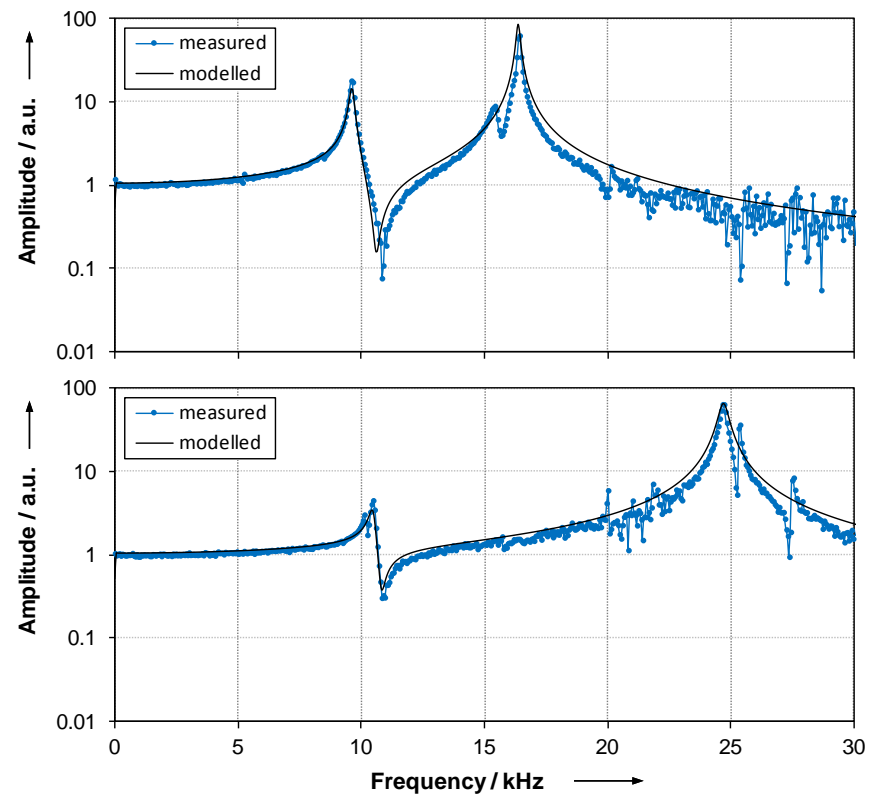

Figure 19. Measured and modelled transducer output in the frequency domain for the shock excitation with an impacting pendulum mass of $7 \mathrm{~g}$, load mass $5 \mathrm{~g}$ (top) and $0.65 \mathrm{~g}$ (bottom), transducer stiffness estimated from sinusoidal excitation measurements.

mass apparently does not show noticeable dynamic effects, the pendulum pulse of $0.2 \mathrm{~ms}$ clearly exhibits signal ringing from the first resonance at about $10 \mathrm{kHz}$ which is correctly described by the model.

Considering the model response of even shorter force pulses, i.e. with excitations of higher frequency content, Figure 18 shows the respective signals of shock force experiments obtained with a pendulum of $7 \mathrm{~g}$ applying load masses of $m_{\mathrm{L}}=0.65 \mathrm{~g}$ and $m_{\mathrm{L}}=5.0 \mathrm{~g}$, respectively. These shock pulses of less than $0.1 \mathrm{~ms}$ strongly excite the transducer's first two axial resonances. The measured and modelled shock responses 
agree fairly well, although the modelled force output is slightly larger. Possible explanations for this discrepancy, which is in the scope of future research, are the uncorrected frequency response of the transducer's electrical signal chain and the influence of the signal filtering and windowing on the model's input force signal. Applying the discrete Fourier transform to the windowed transducer output signal ringing, the corresponding comparison in the frequency domain is shown in Figure 19. The modelled and measured amplitude responses show a good agreement with some slight deviations at higher frequencies.

A rigorous uncertainty evaluation for the parameter estimation is a topic of future research. Given the sensitivity of the different measurement data with respect to changes in the transducer stiffness parameter $k$, we would expect the uncertainty associated to be about $10 \%$. Future research will focus on the statistical evaluation of the influences on the fitting process and the determination of a full uncertainty budget.

\section{CONCLUSIONS AND OUTLOOK}

The approach of a model-based dynamic calibration of force transducers is typically employed to describe the dynamic behaviour of force transducers in a given application, irrespective of the mechanical calibration set-up. However, recent interlaboratory comparisons using sine and shock excitation experiments revealed discrepancies in the reported transducer stiffness parameters. Therefore, new experimental investigations were presented, focusing on measurements with a small strain gauge transducer of high bandwidth. This showed that several challenges have to be solved to identify the model parameters with consistent results independent of the calibration methods used. To this end, a more generalized mechanical model was proposed, which is capable of linking the parameter identification results obtained with different experimental methods to a much better degree than before. The chosen model approach correctly describes the observed resonance behaviour of a force transducer with small and large load masses in shock and sine force calibration measurements. For the remaining differences in the modelled and measured frequency responses, finite element analyses showed that they result from parasitic modes which cannot be incorporated into a uniaxial lumped-mass model. Therefore, future research will focus on improving the determination of the model parameters based on a statistical model for a simultaneous fitting of data sets from different experiments.

\section{ACKNOWLEDGEMENT}

The authors gratefully acknowledge their colleagues at the PTB working group Realization of Acceleration for assisting with the high-frequency sine force measurements. They would also like to thank the company HBM for providing CAD data to model the force transducer selected for this case study.

\section{REFERENCES}

[1] S. Eichstädt, V. Wilkens, A. Dienstfrey, P. Hale, B. Hughes, C. Jarvis, "On challenges in the uncertainty evaluation for timedependent measurements”, Metrologia, 53, 2016, pp. S125-S135.

[2] C. Bartoli et al., "Dynamic calibration of force, torque and pressure sensors", Proc. of Joint IMEKO International TC3, TC5 and TC22 Conference, Feb. 3-5, 2014, Cape Town, South Africa.

[3] EMRP JRP IND09 "Traceable dynamic measurement of mechanical quantities", website http://projects.ptb.de/emrp/ ind09.html.

[4] C. Schlegel, G. Kieckenap, B. Glöckner, A. Buß, R. Kumme, "Traceable periodic force measurement", Metrologia, 49, 2012, pp. 224-235.

[5] N. Medina, J. L. Robles, J. de Vicente, "Realization of sinusoidal forces at CEM", Proc. of Joint IMEKO International TC3, TC5 and TC22 Conference, Feb. 3-5, 2014, Cape Town, South Africa.

[6] V. Nicholas, A. Chijioke, "Traceable dynamic calibration of force transducers by primary means", Metrologia, 53, 2016, pp. S136S148.

[7] M. Kobusch, S. Eichstädt, L. Klaus, T. Bruns, "Investigations for the model-based dynamic calibration of force transducers by using shock excitation”, ACTA IMEKO, ISSN 2221-870X, 4 (2), 2015, pp 45-51.

[8] M. Kobusch, "Influence of mounting torque on the stiffness and damping parameters of the dynamic model of a $250 \mathrm{kN}$ shock force calibration device", 7th Workshop on Analysis of Dynamic Measurements, Oct. 15-16, 2012, Paris, France.

[9] M. Kobusch, S. Eichstädt, L. Klaus, T. Bruns, "Analysis of shock force measurements for the model-based dynamic calibration", 8th Workshop on Analysis of Dynamic Measurements, May 5-6, 2014, Turin, Italy.

[10] D. Kraft, "A software package for sequential quadratic programming”, 1988 Tech. Rep. DFVLR-FB 88-28, DLR German Aerospace Center - Institute for Flight Mechanics, Cologne, Germany.

[11] A. Gelman et al., Bayesian Data Analysis, Third Edition. Chapman and Hall/CRC, 2013, ISBN 978-1-4398-4095-5.

[12] M. Kobusch et al., "Proyecto de investigación europeo para la medición dinámica de magnitudes mecánicas", Proc. of Simposio de Metrología 2014, Oct. 6-10, 2014, Querétaro, México, pp. 111-119. 